\title{
Developing a Scale for Measuring Uncertainty in Patients with Unruptured Intracranial Aneurysms Undergoing Endovascular Coiling
}

\author{
Mitsumi Masuda1, Hidenori Ohishi², Noriko Terunuma ${ }^{3}$, Ikuko Tojima ${ }^{3}$ \\ ${ }^{1}$ Department of Critical Care Nursing, Graduate School of Nursing, Kitasato University, Tokyo, Japan \\ ${ }^{2}$ Department of Medical Science, Graduate School of Medicine, Juntendo University, Tokyo, Japan \\ ${ }^{3}$ Nursing Department, Juntento University Hospital, Tokyo, Japan \\ Email: mitsumi 0323@yahoo.co.jp, ohishi@juntendo.ac.jp, terunuma@juntendo.ac.jp, \\ itojima@juntendo.ac.jp
}

Received 6 September 2015; accepted 26 October 2015; published 29 October 2015

Copyright (C) 2015 by authors and Scientific Research Publishing Inc.

This work is licensed under the Creative Commons Attribution International License (CC BY).

http://creativecommons.org/licenses/by/4.0/

(c) (i) Open Access

\section{Abstract}

A measure called the Uncertainty in Unruptured Intracranial Aneurysm Patients Undergoing Endovascular Coiling Scale (UUIACS) was developed and its validity and reliability were examined. The 49 questions that comprised the original draft of the UUIACS were created based on interview data. Based on data from 172 participants, exploratory and confirmatory factor analyses were conducted. As a result of exploratory factor analysis, the UUIACS e retained 17 items and extracted four factors ("Lack of decision-making cues", "Lack of information and complexity of information interpretation", "The ambiguous nature of the disease", and "The unpredictable living with UIA"). All of the UUIACS items showed adequate internal consistency. Between the UUIAC scale and the Universal Uncertainty in Illness Scale (UUIS), the Health Locus of Control (HLC) scale, and the SF-36v2 ${ }^{\circledR}$ (Japanese version), positive correlations were found between the UUIACS and UUIS, and the HLC scale at a $1 \%$ significance level indicating concurrent validity. According to confirmatory factor analysis, the UUIACS had an acceptable goodness of fit. Given these findings, the UUIACS was judged to have satisfied the criteria for use in a clinical setting, although further investigation was required.

\section{Keywords}

Unruptured Intracranial Aneurysms, Endovascular Coiling, Uncertainty Scale 


\section{Introduction}

Given the increased use of brain scans, cranial MRA/3D-CTA, and other minimally-invasive diagnostic imaging techniques, there has been a sharp increase in patients unexpectedly diagnosed with unruptured intracranial aneurysms (UIA) [1]. In Japan, more than 10,000 individuals a year receive this diagnosis. A UIA can lead to a subarachnoid hemorrhage, for which the fatality rate is extremely high; therefore, it has been recommended that patients meeting the accepted criteria for medical treatment, receive preventative surgery (neurosurgical clipping or cerebral endovascular coiling treatment), with the aim of preventing a rupture [2] [3].

When a UIA is detected, people face an uncertain situation. Beginning from the time before the development of symptoms, an individual is aware of the potential dangers of this illness. People who are asymptomatic and have been diagnosed with a UIA unexpectedly, become aware of its existence with inadequate psychological preparation, and consequently start to show an increase in uncertainty [4] [5], a decline in their quality of life (QOL) [6], and a deterioration in their mental health [7]. Furthermore, people who are currently healthy have difficulty making decisions about treatment for a UIA, such as choosing whether to undergo preventative medical care and choosing among the options of neurosurgical clipping, neurosurgical endovascular coiling, and active observation. A fairly large number of endovascular surgery patients have problems with imperfect closure and recanalization, requiring surgical correction [8] [9]. The results of prior studies [10] suggest that from their first diagnosis until their recovery from treatment, patients undergoing endovascular surgery to correct a UIA may experience emotional distress, such as anxiety and conflict linked to their recognition of uncertainties, such as the ambiguity of late-stage impairment and inadequate dissemination of information about new treatments. Therefore, it is important to assess in detail and with accuracy, the degree of uncertainty that patients with a UIA are aware of over the course of their treatment and recuperation, and to offer assistance aimed at mitigating or removing this uncertainty.

Despite the development of a Japanese version [11] of the Mishel Uncertainty in Illness Scale-Community Form (MUIS-C) [12], which measures uncertainty in illness, and the Universal Uncertainty in Illness Scale to be used for Inpatients and Outpatients (UUIS) [13], a similar measure that can be used regardless of the treatment setting. These linear scales do not reflect this illness's particular discomfort or specific conditions.

Therefore, this study focuses on the general idea of uncertainty concerning the subjective interpretations of responses to illness and medical treatment. A scale is also developed to measure the uncertainty that affects patients who have undergone endovascular surgery to treat a UIA, and to assess the scale's validity and reliability.

\section{Definition of Uncertainty}

The definition of uncertainty in this study is based on Mishel's definition as "the inability to determine the meaning of illness-related events..., the cognitive state created when the person cannot adequately structure or categorize an event because of the lack of sufficient cues" [14].

\section{Methodology}

The name of the measure being developed and evaluated is the Uncertainty in Unruptured Intracranial Aneurysm Patients Undergoing Endovascular Coiling Scale (henceforth referred to as the UUIACS).

\subsection{Initial Development of the UUIACS}

An item pool was compiled with passages describing uncertainty, which had been excerpted from interview data of 31 patients diagnosed with UIA at the Department of Neurosurgery at Metropolitan A University Hospital. The interviewed patients had no previous experience of surgery related to intracranial aneurysms, and were undergoing endovascular coiling for the first time. Questions were developed using existing scales that measure uncertainty: the Mishel Uncertainty in Illness Scale-Community Form (MUIS-A) [15], the MUIS-C Japanese edition [11], and the UUIS [13].

Next, similar and overlapping content were clustered, and 52 questions were developed. The questions were classified based on their meaning and organized into 6 categories: 1) Nature of the disease; 2) The treatment characteristics; 3) Information; 4) Decision making; 5) Course of the future; and 6) Living with UIA [16].

The 52 questions were checked by another researcher, two doctors, two nurses, and four patients who previously underwent endovascular coiling to ensure that the expressions used were appropriate; some revisions 
were made. The questionnaire consisted of 52 items each with five response options (strongly disagree, disagree, undecided, agree, strongly agree) rated on a scale of 1 - 5 points. Pilot tests were conducted with 50 UIA patients. The analysis found that two questions had a ceiling effect (percentage of patients who scored at the ceiling level — that corresponded to $10 \%$ best results on the scale), and one had a floor effect (percentage of patients who scored at floor level—equivalent to the $10 \%$ worst results on the scale); these items were eliminated, leaving the questionnaire with 49 questions. Additionally, a final analysis was performed to confirm the internal consistency and wording of the scale. In the end, the original draft of the UUIACS had 6 categories and 49 questions.

\subsection{Developing the UUIAS-C and Analyzing Its Validity and Reliability}

The survey portion of the UUIACS was distributed to develop it and analyze its validity and reliability. Exploratory factor analysis, internal consistency, validity testing, and confirmatory factor analysis were performed on the survey.

\subsection{Participants}

\subsubsection{Target Group}

The survey was administered at two different institutions to convenience samples of UIA patients who underwent neurosurgery and endovascular coiling as part of their treatment. The candidates in the target group were patients who were undergoing endovascular coiling for the first time and had consented to participate in the study.

\subsubsection{Participants' Characteristics}

Patients' gender, age, smoking behavior, family composition, medical history, and other patient attributes were surveyed via medical examination and patient interviews. The first author performed structured interviews. The content of the question included smoking behavior, family composition, medical history, and circumstances of discovery.

\subsection{Measures}

\subsubsection{Uncertainty}

The original draft of the UUIACS consisted of 6 categories and 49 questions, with responses on a 5-point scale (strongly disagree, disagree, undecided, agree, strongly agree) ranging from 1 - 5 respectively. The total and subscale scores were calculated by simply summing the scores, a higher score indicated a higher degree of uncertainty experienced.

\subsubsection{Scales Used for Assessing Criterion-Related Validity}

\section{1) Universal Uncertainty in Illness Scale (UUIS)}

The UUIS, developed by [13] to measure uncertainty, is a linear scale composed of 26 items and 6 subscales. The scale can be used regardless of whether the medical treatment is administered on an inpatient or outpatient basis, and it better reflects how Japanese patients experience uncertainty in relation to their illness. A higher score indicates a higher level of uncertainty.

\section{2) Health Locus of Control Scale (HLC Scale)}

The HLC scale [17] measures subjective feelings of control over one's health. Health Locus of Control focuses on whether or not one believes that there is a cause associated with an event resulting from one's own behavior related to health and illness, and whether one believes that one had control over its cause. Questions 26-30 clearly relate to uncertainty [18] [19]. The scores for the HLC Scale range from 11 to 33 points, with a higher score indicating stronger subjective feelings of control over one's health.

\section{3) Short Form-36v2 ${ }^{\circledR}$ Health Survey-Japanese Version (SF-36v2 ${ }^{\circledR}$ )}

The SF-36v2 ${ }^{\circledR}$ survey [20] [21], which measures health-related quality of life, SF-36v2 ${ }^{\circledR}$, consists of items related to physical functioning, everyday role functioning (physical), bodily pain, overall health, vitality, social functioning, everyday role functioning (mental), and eight subscales measuring mental health. The score is converted to a 0-100-point scale, and a higher score indicates a higher quality of life. 


\subsection{Survey Method}

A questionnaire survey method was used. The researchers explained the intent of the survey and any ethical considerations to the patients. After obtaining informed consent, the questionnaires were distributed and the participants' responses were requested. The responses were collected on the same day, or they were returned by mail. The data collection was conducted from July 2013 to March 2014.

\subsection{Data Analyses Methods}

The following analyses were performed using statistics software SPSS 21.0J and Amos 22.0J. Item analysis was used to examine ceiling and floor effects and item-total correlations. Construct validity was tested by comparing the 6 categories that were developed when composing the original draft with the factors that were extracted from exploratory factor analysis. Criterion-related validity was tested by analyzing the correlation coefficients using UUIS, the HLC scale, and the Japanese version of the SF36v2 ${ }^{\circledR}$. Internal consistency was assessed by calculating Cronbach's alpha coefficients for the entire scale and each subscale. The model's degree of conformance was examined using confirmatory factor analysis, which was performed on the latent variables that included the items of the UUIACS that were selected as factors as a result of exploratory factor analysis. The UUIACS model's degree of conformance was tested using the goodness of fit index (GFI), degree-of-freedom adjusted goodness of fit index (AGFI), comparative fit indicator (CFI), and root mean square error of approximation (RMSEA).

\subsection{Ethical Considerations}

This study was conducted with the approval of the medical ethics committees of the institutions that implemented the survey in June 2013. The participants gave their consent after the study's intent was explained to them. At that time, the freedom of the participants to withdraw from the study and the protection of their anonymity were explained verbally and in writing, after which signatures were obtained indicating their consent. Their responses to the questionnaire were collected on the same day, and when this was not possible, the questionnaire was taken home and returned by mail.

\section{Results}

\subsection{Clinical Research Settings and Data Collection Procedures}

A request for cooperation was made to two institutions, both of which agreed. Questionnaires were distributed to 201 participants and 173 were retrieved (68.1\% return rate); 10 of the returned questionnaires had missing responses; 9 of them were missing fewer than $10 \%$ of the total responses. Therefore, the total score was adjusted to reflect the average of the UUIACS, UUIS, HLC scale, and SF-36v2 ${ }^{\circledR}$ (Japanese version). One of the returned questionnaires was missing more than $10 \%$ of the total responses and was eliminated from the study. Valid responses from 172 patients were used in the analysis of the data (response rate: 99.4\%).

\subsection{Participants}

As shown in Table 1, the participants consisted of 54 men and 118 women, with an average age of 62.3 (SD = 10.9); 44 participants had a family history of subarachnoid haemorrhage and 128 did not; 55 were smokers, and 117 were not.

\subsection{Item Analysis}

The item-total correlations ranged from 0.22 to 0.64 ; none of the questions had a correlation coefficient below 0.2 ; therefore, none of them were trimmed. Descriptive statistics were calculated and 15 questions were eliminated: 8 questions had ceiling effects (e.g. "I do not know the cause of my intracranial aneurysm") and 7 questions had floor effects (e.g. "I have not decided whether to continue medical examination going forward").

\subsection{Factors Selected through Exploratory Factor Analysis}

Exploratory factor analysis using promax rotation, was conducted on 34 questions (after eliminating 15 questions) (Table 2). A scree plot using principal component analysis showed that there were 4 factors, and after 
Table 1. Demographic characteristics of participants $(n=172)$.

\begin{tabular}{cccc}
\hline \multirow{2}{*}{ Age } & & Number & Proportion (\%) \\
\hline \multirow{2}{*}{ Sex } & Mean \pm SD & $62.3 \pm 10.9$ & \\
& Male & 54 & 31.4 \\
Employed & Female & 118 & 68.6 \\
& Yes & 99 & 57.6 \\
High blood pressure & No & 73 & 42.4 \\
& Yes & 82 & 47.7 \\
Smoker & No & 90 & 52.3 \\
& Yes & 55 & 32.0 \\
Drinks alcohol & No & 117 & 68.0 \\
& Yes & 62 & 36.0 \\
Family history & No & 110 & 64.0 \\
& Yes & 44 & 25.6 \\
Circumstances of discovery & No & 128 & 74.4 \\
& Screening for UIA & 123 & 71.5 \\
Size & Symptoms & 49 & 28.5 \\
& Mean \pm SD & $4.9 \pm 2.1$ & \\
& 5 mm or more & 69 & 40.1 \\
& Less than 5 mm & 103 & 59.9 \\
\hline
\end{tabular}

${ }^{\mathrm{a}}$ Alternate option for UIA size was $>5 \mathrm{~mm}$.

eliminating questions with a factor loading of less than 0.4, as shown in Table 2, 4 factors and 17 questions achieved a valid factor structure and were extracted for use in the UUIACS. The cumulative contribution ratio before rotation was $45.85 \%$, and the Spearman's correlations between the four factors ranged from $0.36-0.60$, all of them having significant positive correlations.

\subsection{Subscale Naming}

The contents of the items selected for each of the four extracted factors were interpreted. The first factor (6 items) pertained to uncertainty concerning the complexity of decision making (e.g. "I do not know which to choose, endovascular surgery or craniotomy" and "I do not know whether it is good that the unruptured intracranial aneurysm was detected"). This factor was named "Lack of decision-making cues". The second factor (5 items) pertained to uncertainty about the lack of information and the interpretation of information (e.g. "I feel like the more I learn about my illness and treatment, the less I understand and the more questions mount up" and "I do not know what other people with the same illness may be experiencing"). This factor was named "Lack of information, complexity of information interpretation". The third factor (3 items) pertained to uncertainty about the inability to specify the nature of the illness, as it was discovered at an asymptomatic stage before a rupture (e.g. "I do not know whether or not an unruptured intracranial aneurysm is an illness"). This factor was named "The ambiguous nature of the disease". The fourth factor (3 items) pertained to uncertainty about living with a UIA because it is a relatively recent illness (e.g. "Unruptured intracranial aneurysm is an illness with which I am not very familiar"). This factor was named "The unpredictable living with UIA".

\subsection{Assessment of Reliability (Internal Consistency)}

Cronbach's alpha for the overall UUIACS (17 questions) was 0.84 , and for each of the four factors, it ranged from 0.61 - 0.82 (Table 2).

\subsection{Assessment of Validity}

\subsubsection{Assessment of Construct Validity}

The items that fell under the four factors extracted through exploratory factor analysis (1) "Lack of decision- 


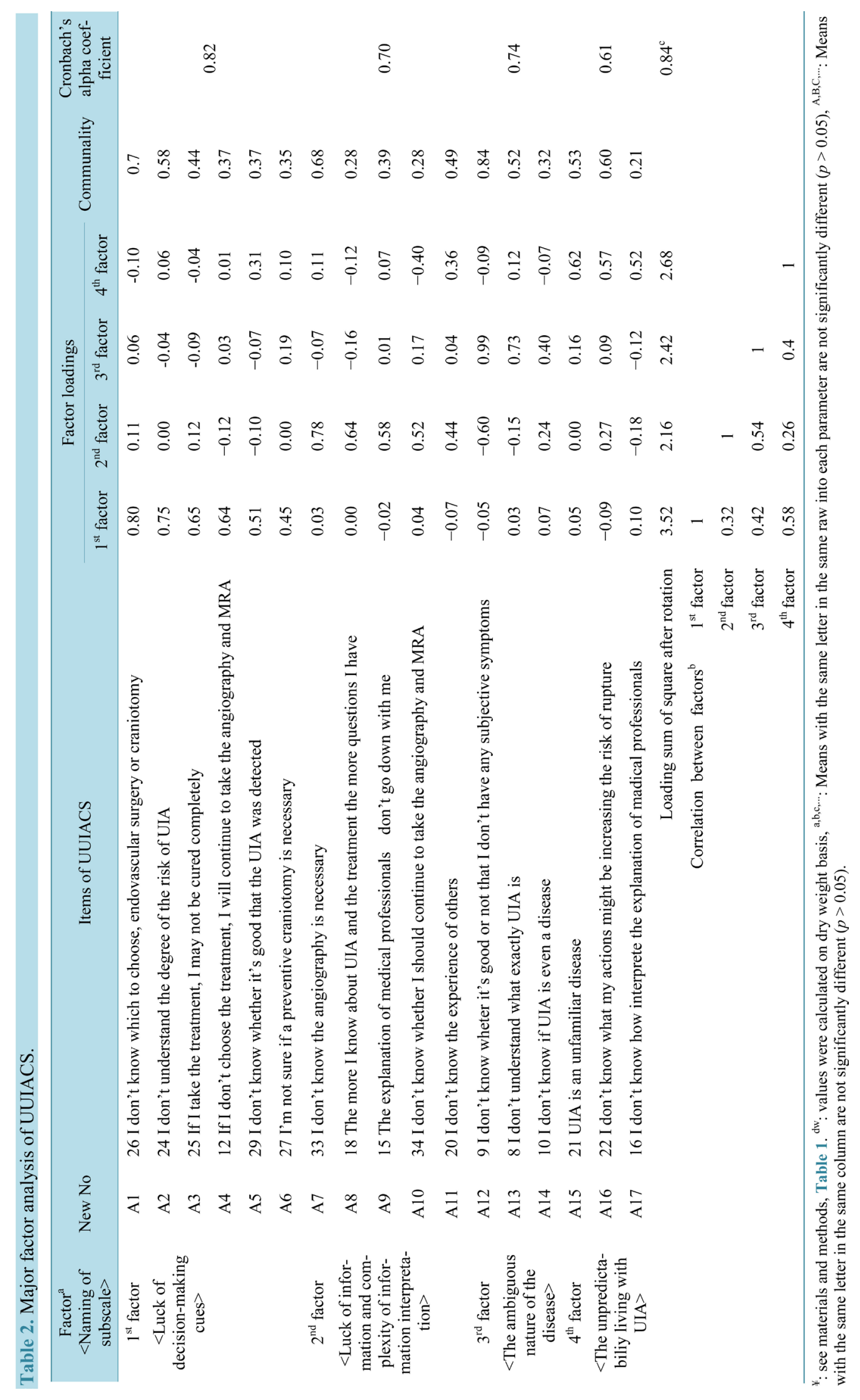


making cues"; 2) "Lack of information, complexity of information interpretation”; 3) "The ambiguous nature of the disease"; and 4) "The unpredictable living with UIA") were compared with the items that were classified under the six categories derived from the content analysis when creating the original draft of the questionnaire 1) "Nature of the disease"; 2) "The treatment characteristics"; 3) "Information"; 4) "Decision making”; 5) "Course of disease"; and 6) "Living with a UIA"). The results showed that the factor, "Lack of decision-making cues" consisted of the same items as categories 2) and 4). "Lack of information, complexity of information interpretation" consisted of the same items as category 3). "The ambiguous nature of the disease" consisted of the same items as category 1) and "The unpredictable living with UIA" consisted of the same items as categories 5) and $6)$.

\subsubsection{Assessment of Criterion-Related Validity}

To assess criterion-related validity, Spearman's correlations were calculated between the total UUIACS score, and the scores on the UUIS, HLC scale, and the SF-36c2 ${ }^{\circledR}$ Japanese edition. As shown in Table 3, the analyses yielded a positive correlation of $r=0.65$ with UUIS, and a negative correlation of $r=-0.25$ with the HLC scale, that was significant at the 0.01 level. A problem remained with the SF-36v2 ${ }^{\circledR}$ (Japanese version), which had a negligible correlation of $r=-0.14$.

\subsection{Examination of the Model's Goodness of Fit}

The goodness of fit of the hypothetical model obtained through exploratory factor analysis was assessed through confirmatory factor analysis. If the four factors are treated as latent variables, this should result in a GFI of 0.90 and an AGFI of 0.85, satisfying the goodness of fit criteria of a GFI of 0.9 or above, an AGFI of 0.85 or above, with the GFI greater than the AGFI. The GFI of 0.92 in this study, satisfied the criterion that the GFI should be above 0.9 , and the RMSEA of 0.07 satisfied the criterion of 0.08 . Between the latent variables and the observed variables, as shown in Figure 1, all of the items achieved a valid path coefficient of 0.50 or above.

\section{Discussion}

This study attempted to develop a scale to measure the uncertainty that affects patients undergoing endovascular surgery for a UIA, focusing on uncertainty as a general concept concerning the subjective interpretation of illness and treatment. The result was the creation of the UUIACS, composed of 17 items and 4 subscales.

\subsection{The Reliability and Validity of the UUIACS}

Regarding the reliability of the UUIACS, Cronbach's alpha for the overall scale (17 questions) was 0.84, and ranged from 0.61 to 0.82 for each of the four subscales. Factors 1-3 exceeded 0.7. Only the fourth factor, at 0.61 , fell below the criterion of 0.7 . This finding could be caused by fewer questions. However, the Cronbach's alpha of 0.84 for the overall scale exceeded the criterion; therefore, it can be considered a generally reliable measure.

The scale's content validity was conducted the experts panel, criterion-related validity was tested by analyzing the correlation coefficients using UUIS, the HLC scale, and the Japanese version of the SF36v2 ${ }^{\circledR}$, and construct validity were examined by comparing 6 categories of the original draft from exploratory factor analysis. Content validity was examined during the creation of the original UUIACS draft, with consideration of whether or not the instrument had been adequately conceptualized to encompass the entire field, as understood from the literature review conducted as part of a qualitative study. The items of the UUIACS inquire about the uncertain-

\begin{tabular}{ccccc} 
Table 3. Criterion-related validity $(\mathrm{n}=172)$. \\
\hline Scale & \multicolumn{4}{c}{ Spearman's correlation coefficients } \\
\cline { 2 - 5 } & UUIACS total score & UUIS & HLC scale & SF-36v2 $^{\circledR}$ \\
\hline UUIACS total score & - & & & \\
UUIS & $0.65^{* *}$ & - & - & \\
HLC scale & $-0.25^{* *}$ & $-0.15^{*}$ & - & - \\
SF-36v2 $2^{\circledR}$ & -0.14 & -0.06 & $0.17^{*}$ & \\
${ }^{* *} p<0.01,{ }^{*} p<0.05$. & & & &
\end{tabular}




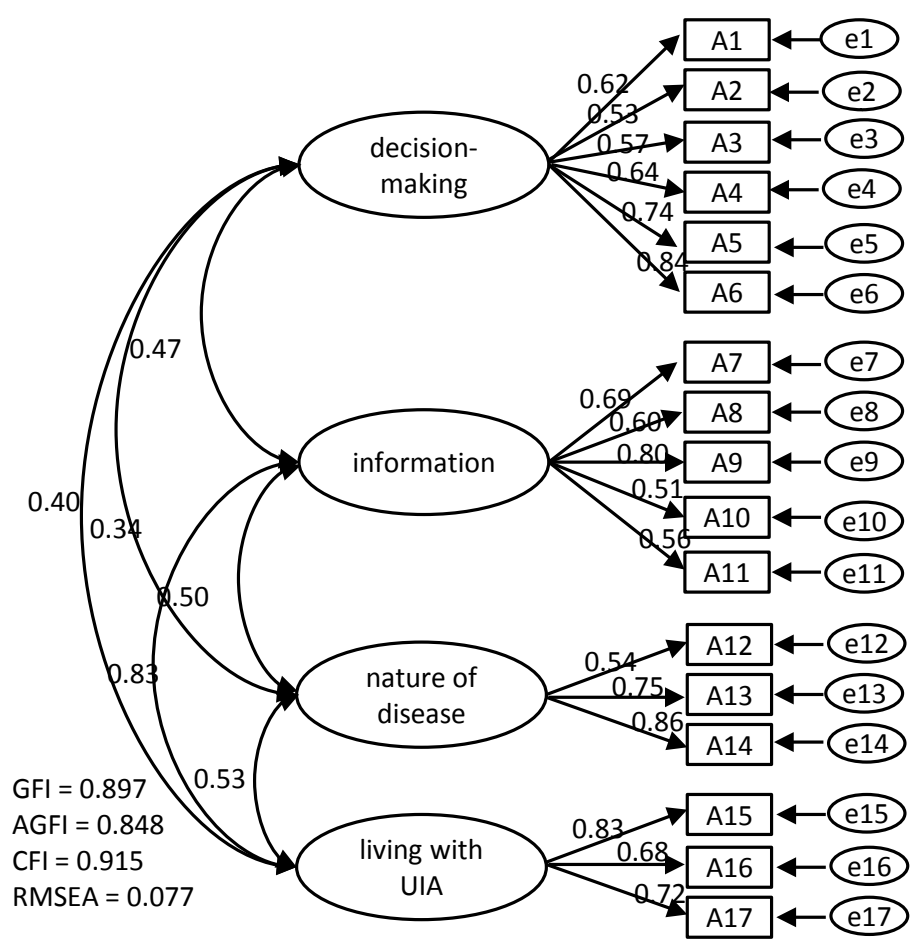

Figure 1. Test of goodness of fit.

ty of patients who undergo endovascular coiling for an UIA. The scale is not only for specialists who have received university degrees and are conversant in the development of linear scales; it also is of practical value for obtaining opinions from doctors and nurses who examine and treat large numbers of UIA patients, as well as the UIA patients who experience this type of uncertainty.

Regarding the scale's criterion-related validity, a strong positive correlation was observed between the total UUIACS score and the UUIS score. A significant negative correlation, although weak, was observed with the HLC scale score, confirming a degree of concurrent validity. However, there remains the issue of revising the scale because of the lack of a correlation with the SF-36 $\mathrm{v2}^{\circledR}$ (Japanese version) and this issue will influence the future studies that are conducted.

In terms of the scale's construct validity, the 6 categories that were extracted through content analysis by the researchers during the original drafting stage of the UUIACS had an almost identical structure to the four factors that were extracted through exploratory factor analysis. Before rotation, the four factors' cumulative contribution ratio was $45.85 \%$. Consequently, the UUIACS can be considered to have construct-related validity. Based on this finding, we can that the UUIACS is a scale with adequate validity and reliability, and may be used in clinical settings because it satisfied the criteria for the model's goodness of fit through confirmatory factor analysis.

\subsection{Significance of the UUIACS'S Characteristics and Development}

The UUIACS, a linear scale measuring uncertainty, consists of 17 items in 4 subscales. The significance and characteristics of this scale can be discussed in comparison with the MUIS, which has been used around the world and with the UUIS, which was developed in Japan.

Mishel's scale was developed using American patient data (Mishel 1981) [15]. However, the UUIACS was based on Japanese patient data, so it might be a more accurate measure of how uncertainty is recognized by patients with a Japanese cultural background. Furthermore, the UUIS, has overcome problems with mis-translations and word nuances that affected the use of Mishel's scale [15] with Japanese people. It can be used regardless of treatment site, whether a patient is hospitalized or recuperating at home on an outpatient basis, so it is particularly useful for measuring uncertainty on an ongoing basis. The UUIACS was developed based on a longitudinal qualitative study, and reflects the specific phenomenon of UIA patients undergoing endovascular coiling; there- 
fore, it can be used continuously, as a measure that is highly relevant to this medical specialty. For this reason, there is a greater chance of acquiring a better understanding of the characteristics and changes of patients with this illness.

Moreover, the MUIS and UUIS measure the uncertainty recognized by patients while they are ill. However, UIA patients often express that "it is unclear whether an unruptured intracranial aneurysm is an illness", as the UIA is discovered at an asymptomatic stage; hence, this creates a key discrepancy between the MUIS and UUIS, which assume that the respondent must be experiencing the illness. Furthermore, UIA is a relatively new illness, and endovascular surgery is a relatively new treatment. Although making decisions regarding medical treatment is extremely difficult, the MUIS and UUIS do not include the concept of complex decision-making. Further, the UUIACS better reflects the nature and source of the uncertainties characteristic of UIA patients, and may help in the development of policies that remove the uncertainty faced by patients undergoing endovascular coiling to treat a UIA, or mitigate its effects.

\subsection{Potential Use of the UUIACS to Evaluate Nursing Support}

The UUIACS may be used to assess the uncertainty that UIA patients experience during recovery. For this reason, it can be used as an assessment tool for nurses. Furthermore, it is useful for examining how nurses might set goals and provide concrete support to patients by understanding their source, nature, and degree of uncertainty. For instance, when dealing with the lack of information or ambiguous subjective symptoms due to uncertainty over disease characteristics, uncertainty can eased and transformed into certainty by providing information, explaining whether subjective symptoms are due to UIA or not. On the other hand, with regard to decision making, uncertainty will not necessarily be transformed into certainty, most helpful information. In such cases, teaching probabilistic thinking skills helps patients to at least partially accept this certainty. Using the UUIACS, together with the HLC scale and a depression scale, may enable nurses to evaluate the results of their nursing support.

\section{Study Limitations and Recommendations for Future Research}

The UUIACS was developed through a region-based study that experienced difficulty guaranteeing an adequate number of participants. More than half of the scale's questions had to be eliminated during the item and factor analysis stages of its development; yet, problems with the expression of items remained. There was almost no correlation between the scale and the Japanese version of the SF-36v2 ${ }^{\circledR}$, and this finding will determine future plans for the scale's further development.

\section{Conclusion}

The development of a scale that can reflect the uncertainty experienced by patients undergoing endovascular surgery due to UIA is attempted. The result of this endeavor is a scale consisting of 17 items in 4 factors named, "Lack of decision-making cues", "Lack of information and complexity of information interpretation", "The ambiguous nature of the disease", and "The unpredictable living with UIA". The validity and reliability of the UUIACS are verified by assessing the scale's criterion-related validity and internal consistency.

\section{Acknowledgements}

The authors acknowledge that this study received funding assistance from JSPS KAKENHI Grant Number 24890253.

The authors are grateful to the subjects of this study who provided us with invaluable information. They would also like to thank the outpatient nurses, senior staff, doctors, and nurses of the hospital ward.

\section{Conflict of Interest}

The authors have no financial conflicts of interest to disclose concerning the research.

\section{References}

[1] Jung, Y.J., Ahn, J.S., Park, E.S., Kwon, D.H., Kwun, B.D. and Kim, C.J. (2011) Surgical Results of Unruptured Intracranial Aneurysms in the Elderly: Single Center Experience in the Past Ten Years. Journal of Korean Neurosurgical 
Society, 49, 329-333. http://dx.doi.org/10.3340/jkns.2011.49.6.329

[2] Sonobe, M., Yamazaki, T., Yonekura, M. and Kikuchi, H. (2010) Small Unruptured Intracranial Aneurysm Verification Study: SUAVe Study, Japan. Stroke, 41, 1969-1977. http://dx.doi.org/10.1161/STROKEAHA.110.585059

[3] UCAS Japan Investigators (2012) The Natural Course of Unruptured Cerebral Aneurysms in a Japanese Cohort. New England Journal of Medicine, 366, 2474-2482. http://dx.doi.org/10.1056/NEJMoa1113260

[4] Otawara, Y., Ogasawara, K., Kubo, Y., Tomitsuka, N., Watanabe, M., Ogawa, A., Yamadate, K., et al. (2004) Anxiety before and after Surgical Repair in Patients with Asymptomatic Unruptured Intracranial Aneurysm. Surgical Neurology, 62, 28-31. http://dx.doi.org/10.1016/j.surneu.2003.07.012

[5] van der Schaaf, I.C., Brilstra, E.H., Rinkel, G.J.E., Bossuyt, P.M. and van Gijn, J. (2002) Quality of Life, Anxiety, and Depression in Patients with an Untreated Intracranial Aneurysm or Arteriovenous Malformation. Stroke, 33, 440-443. http://dx.doi.org/10.1161/hs0202.102335

[6] Yamashiro, S., Nishi, T., Koga, K., Goto, T., Kaji, M., Muta, D., Fujioka, S., et al. (2007) Improvement of Quality of Life in Patients Surgically Treated for Asymptomatic Unruptured Intracranial Aneurysms. Journal of Neurology, Neurosurgery \& Psychiatry, 78, 497-500. http://dx.doi.org/10.1136/jnnp.2006.098871

[7] Brilstra, E.H., Rinkel, G.J.E., van der Graaf, Y., Sluzewski, M., Groen, R.J., Lo, R.T.H. and Tulleken, C.A.F. (2004) Quality of Life after Treatment of Unruptured Intracranial Aneurysms by Neurosurgical Clipping or by Embolisation with Coils. Cerebrovascular Diseases, 17, 44-52. http://dx.doi.org/10.1159/000073897

[8] Cheong, J.J., Ghinea, N. and van Gelder, J.M. (2004) Estimating the Annual Rate of de Novo Multiple Aneurysms: Three Statistical Approaches. Neurosurg Focus, 17, 1-8. http://dx.doi.org/10.3171/foc.2004.17.5.8

[9] Tsutsumi, K., Ueki, K., Morita, A. and Kirino, T. (2008) Risk of Aneurysm Recurrence in Patients with Clipped Cerebral Aneurysms: Results of Long-Term Follow-Up Angiography. Stroke, 39, 1276-1285.

[10] Masuda, M. and Inoue, T. (2010) Experience of Uncertainty in Patients with Unruptured Intracranial Aneurysms Treated with Endovascular Coiling and Their Nursing Care. Journal of Japan Academy of Critical Care Nursing, 6, 16-25. http://dx.doi.org/10.11153/jaccn.6.3_16

[11] Nogawa, M. (2004) Study on the Reliability and Validity of the Japanese-Language Version of the Mishel Uncertainty in Illness Scale-Community Form. Japan Journal of Nursing Science, 24, 39-48. http://dx.doi.org/10.5630/jans1981.24.3_39

[12] Mishel, M.H. and Epstein, D. (1997) Uncertainty in Illness Scales Manual. University of North Carolina, Chapel Hill.

[13] Nogawa, M. (2012) Development of a Universal Uncertainty in Illness Scale to Be Used for Inpatients and Outpatients. Japan Journal of Nursing Science, 32, 3-11. http://dx.doi.org/10.5630/jans.32.1_3

[14] Mishel, M.H. (1988) Uncertainty in Illness. Journal of Nursing Scholarship, 20, 225-232. http://dx.doi.org/10.1111/j.1547-5069.1988.tb00082.x

[15] Mishel, M.H. (1981) The Measurement of Uncertainty in Illness. Nursing Research, 30, 258-263. http://dx.doi.org/10.1097/00006199-198109000-00002

[16] Masuda, M., Ohishi, H. and Yamamoto, M. (2014) Uncertainty in Patients with Unruptured Intracranial Aneurysms Undergoing Endovascular Surgery: A Qualitative and Inductive Study. Nursing Research, 63, 366-374. http://dx.doi.org/10.1097/NNR.0000000000000050

[17] Wallston, K.A. and Wallston, B.S. (1981) Health Locus of Control Scale. In: Lefcourt, H.M., Ed., Research with the Locus of Control Construct, Academic Press, New York, 15-63. http://dx.doi.org/10.1016/b978-0-12-443201-7.50010-5

[18] Edwards, J.A. and Weary, G. (1996) Antecedents of Causal Uncertainty and Perceived Control—A Prospective Study. European Journal of Personality, 12, 135-148. http://dx.doi.org/10.1002/(SICI)1099-0984(199803/04)12:2<135::AID-PER301>3.0.CO;2-Y

[19] Mast, M.E. (1995) Adult Uncertainty in Illness-A Critical Review of Research. Scholarly Inquiry for Nursing Practice: An International Journal, 9, 3-24.

[20] SF-36v2 ${ }^{\circledR}$ Health Survey (C) 1992, 2000, 2003 Quality Metric Incorporated, Medical Outcomes Trust and Shunichi Fukuhara.

[21] Fukuhara, S. and Suzukamo, Y. (2004) Manual of SF-36v2 Japanese Version. Institute for Health Outcomes \& Process Evaluation Research, Kyoto. 